\title{
Capacity of Mentha spicata (spearmint) Extracts in Alleviating Hormonal and Folliculogenesis Disturbances in a Polycystic Ovarian Syndrome Rat Model
}

\author{
Sanaz Alaee ${ }^{1}$, Mohammad Jafar Bagheri ${ }^{2}$, Mahmood Sadeghi Ataabadi ${ }^{1,3}$, and Farhad Koohpeyma ${ }^{4}$ \\ ${ }^{I}$ Department of Reproductive Biology, School of Advanced Medical Sciences and Technologies, Shiraz University of Medical Sciences, Shiraz, Iran \\ ${ }^{2}$ Department of Anatomical Sciences, Medical Sciences Faculty, Tarbiat Modares University, Tehran, Iran \\ ${ }^{3}$ Student Research Committee, Shiraz University of Medical Sciences, Shiraz, Iran \\ ${ }^{4}$ Department of Endocrinology, Endocrinology and Metabolism Research Center, Shiraz University of Medical Sciences, Shiraz, Iran \\ *Corresponding author's Email: alaee@sums.ac.ir; (DORCiD: 0000-0003-4490-8830
}

\begin{abstract}
Polycystic ovary syndrome, a common cause of infertility among women in the reproductive age, is associated with high levels of androgens. Recognizing the anti-androgenic effects of spearmint, the present study aimed to evaluate the effects of its hydroalcoholic extract on the levels of luteinizing hormone, follicle-stimulating hormone, and testosterone and ovarian folliculogenesis in normal and letrozole-induced polycystic ovary syndrome rats. Female mature rats were divided into six groups ( $\mathrm{n}=8$ per group), as follows: Normal rats (I or Control), normal rats which received $250 \mathrm{mg} / \mathrm{kg}$ spearmint extract (II) or $500 \mathrm{mg} / \mathrm{kg}$ spearmint extract (III), and PCOS-induced rats (IV), PCOSinduced rats which received $250 \mathrm{mg} / \mathrm{kg}$ spearmint extract $(\mathrm{V})$, or $500 \mathrm{mg} / \mathrm{kg}$ spearmint extract (VI). At the end of the experiment the animals were euthanized, and then mentioned parameters were evaluated. Administration of spearmint extract to PCOS rats resulted in a decrease of body weight and testosterone level, higher corpus luteum, and lower ovarian cysts and atretic follicles, compared to PCOS rats which received no spearmint. Accordingly, the spearmint can attenuate polycystic ovarian syndrome-related problems, such as a high testosterone level and ovarian cysts.
\end{abstract}

Keywords: Folliculogenesis, Mentha spicata, Ovary, PCOS, Rat

\section{INTRODUCTION}

Polycystic ovary syndrome (PCOS) is a common hormonal disorder among women in the reproductive age. It has been demonstrated that genetic factors, hormonal disorders, lifestyle, environmental factors and stress contribute to the development of this syndrome (Shaikh et al., 2014a; Krishnapillai et al., 2015). PCOS is often associated with a high level of androgen hormones, obesity, insulin resistance and oligomenorrhea or anovulation (Hatirnaz et al., 2015). Since a high level of androgens is considered the essential factor in PCOS, the animal model of PCOS is frequently created by androgenizing of animals (Van Houten and Visser, 2014). Medicinal plants have been traditionally used as natural medications, and played vital roles in disease prevention and their promotion (Monsefi and Masudi, 2014; Sharangouda et al., 2015; Namavar Jahromi et al., 2019). Mentha spicata, known as spearmint, is a medicinal plant which in Iran's traditional medicine is mainly recommended for digestive system disorders as a carminative and antispasmodic agent, and also for alleviating hirsutism and menstrual pain (Vejdani et al., 2006). The antioxidant, anticancer, antiinflammatory, antifungal, antimicrobial, and antidiabetic properties of Mentha spicata have been shown in some studies (Guimarães et al., 2011; Alaee et al., 2016).

It is known that spearmint is beneficial in decreasing free testosterone level and hirsuitism in women with mild hirsutism with PCOS, and its adverse histopathological effects on kidney, liver and uterine tissue in animals were observed (Akdogan et al., 2003; Akdogan et al., 2004; Akdogan et al., 2007; Guney et al., 2006; Grant, 2010). The effects of this herbal plant on folliculogenesis of ovarian tissue in normal and PCOS conditions were not determined. Present study was designed to evaluate the effects of spearmint extract on follicle stimulating hormonr (FSH), luteinizing hormone (LH), testosterone hormones and ovarian folliculogenesis in the animal model of PCOS induced by letrozole.

\section{MATERIALS AND METHODS}

Preparation of Mentha spicata hydroalcoholic extract

Mentha spicata was purchased from Pursina Pharmaceutical Company, Tehran, Iran. Hydroalcoholic extract was prepared using the maceration method (Monsefi et al., 2015). The dried leaves of the plant were powdered and 
macerated in ethanol for 3 days. Then the solution from the total extract was filtered with filter paper, concentrated by evaporation and stored in refrigerator until being used for the experiments. The yield (w/w) of the solution was $13 \%$ $(\mathrm{g} / \mathrm{g})$.

\section{Animals}

Forty-eight mature Wistar albino female rats were obtained from animal house of Shiraz University of Medical Sciences, Shiraz, Iran. Prior to use in the study, rats were kept in cages in temperature-controlled rooms with constant humidity and $12 \mathrm{hr} / 12 \mathrm{hr}$ light/dark cycle with free access to standard diet and water. For selection of rats with normal estrus cycle, daily vaginal smears were carried out and immediately evaluated with a light microscope (Monsefi et al., 2013). Wistar female rats with two normal estrus cycles were weighed and allocated into the six groups (n: 8) as below; Group I (control): Received $1 \mathrm{ml}$ distilled water orally for 20 days; Group II: Received spearmint extract (250 mg/kg) for 20 days; Group III: Received spearmint extract $(500 \mathrm{mg} / \mathrm{kg}$ ) for 20 days; Group IV: Received letrozole orally for 28 days; Group V: Received letrozole orally for 28 days, and then received spearmint extract (250 mg/kg) for $20 \mathrm{days}$; Group VI: Received letrozole orally for 28 days, and then received spearmint extract (500 mg/kg) for 20 days. Polycystic ovary syndrome induction was carried out by treating rats daily with letrozole (Femara®, made by Novartis Pharmaceuticals Corp., Basel, Switzerland) orally (1 mg/kg) for 28 days, and confirmed by persistent estrus phase and high number of ovarian cysts in ovarian sections via hematoxylin and eosin staining (Neisy et al., 2019).

After treatment duration, animals were weighed, euthanized by inhalation of ether, and a blood sample was taken for hormonal analysis. In addition, the ovarian tissues of all rats were removed and prepared for the histological evaluation (Sadeghi et al., 2017).

\section{Hormonal assay}

Blood samples were collected from the heart, and were centrifuged at $3000 \mathrm{rpm}$ for $15 \mathrm{~min}$. Serum portions were separated and frozen until being evaluated. Serum concentrations of testosterone (Padtan Elm Company, Tehran, Iran), LH and FSH were measured with their specific kits (Hangzhou Eastbiopharm Co., Ltd., Hangzhou, China) (Sadeghi et al., 2017).

\section{Histological analysis}

Ovarian tissues were removed, fixed in $10 \%$ buffered formalin solution, and the paraffin blocks were prepared. The blocks were sectioned at $5 \mu \mathrm{m}$ thickness, and were stained in the hematoxylin and eosin method (Alaee et al., 2014). The number of primordial follicles, primary follicles, secondary follicles, Graafian follicles, atretic follicles, corpus luteum and cysts were counted in ovarian sections using a light microscope (Olympus, Japan).

\section{Statistical analysis}

Statistical analysis was performed using SPSS 16 software (IBM, Armonk, USA). For data analysis, the One-Way ANOVA test was used, followed by the Tukey test to compare the means. P value of $\leq 0.05$ was considered statistically significant.

\section{Ethical approval}

The study protocol was approved by the Animal Ethical Committee of Shiraz University of Medical Sciences (IR.SUMS.REC.1396.S1066), and was carried out in accordance with the university's Guideline for the Care and Usage of Laboratory Animals.

\section{RESULTS}

The results are presented in two parts; A: Evaluation of the effects of spearmint extract on normal female rats (comparing groups I, II and III). At the end of the experiment, the body weight of the animals that received spearmint extract was not different from that of the control group ( $p>0.05$, table 1). The level of LH, FSH and testosterone did not change among spearmint extract-administered groups in comparison to the control group ( $\mathrm{p}>0.05$, table 1). Administration of spearmint extract in two doses of 250 and $500 \mathrm{mg} / \mathrm{kg}$ (groups II and III) led to a significant decrease in the number of primordial follicles $(\mathrm{P}<0.001)$. In addition, the number of primary follicles, secondary follicles, Graafian follicles and corpus luteum decreased in these groups compared to the control group, but it was not statistically significant $(\mathrm{p}>0.05)$. However, the number of atretic follicles was significantly higher in the groups administered spearmint extract compared to the control group ( $<<0.001$, table 2). B: Evaluation of the effects of spearmint extract on PCOS-induced rats comparing with groups C (control), IV, V and VI. At the end of the study, the body weight in the PCOS-induced group was significantly higher than those in the control group (P: 0.031). 
At the end of the experiment, in both PCOS-induced groups that received spearmint extract, the rats' weights were not different from that of the control group $(\mathrm{P}>0.05)$. In the PCOS-induced group that was administered high doses of spearmint extract, weights were significantly less in comparison to those of PCOS-induced rats (P: 0.001, table 1). The level of LH and FSH showed no significant alteration among control, PCOS-induced groups and PCOS-induced groups that received spearmint extract $(\mathrm{p}>0.05)$, but the level of testosterone in the PCOS-induced group was significantly higher in comparison to the control group ( $\mathrm{p}<0.001$ ). In PCOS-induced groups that took two doses of spearmint extract, testosterone level was significantly lower in comparison to the PCOS-induced group ( $\mathrm{p}<0.001$, table 1$)$.

The number of primordial follicles was significantly lower in the PCOS-induced group, and in the PCOS-induced groups which received spearmint extract compared to control group $(\mathrm{P}<0.001)$. The number of primary follicles was not different in PCOS-induced rats by comparing to the control group and the PCOS-induced rats which took spearmint extract $(\mathrm{P}>0.05)$. The number of secondary follicles was meaningfully lower in the PCOS-induced group and those which received spearmint extract as compared to the control group (P: 0.033). There were no Graafian follicles in the PCOS-induced group, and in those which had spearmint extract, but this was not statistically significant comparative to the control group $(\mathrm{P}>0.05)$. The number of atretic follicles and cysts was considerably higher in the PCOS-induced rats in contrast to the control group ( $\mathrm{P}<0.001)$, and in the PCOS-induced group compared to PCOS-induced groups which received spearmint extract $(\mathrm{P}<0.001)$ (Figure 1). The number of corpus lutea in PCOS-induced rats was also meaningfully lower than the control group (P: 0.001), and its number increased significantly in PCOS-induced rats who took the spearmint extract $(250$ and $500 \mathrm{mg} / \mathrm{kg})$ in comparison to PCOS-induced rats $(\mathrm{P}<0.001)$.

Table 1. Body weight at the beginning and end of the experiments, and the level of luteinising hormone, follicle stimulating hormone and testosterone of female rats in studied groups

\begin{tabular}{lccccc}
\hline Groups & $\begin{array}{c}\text { Weight at } \\
\text { the beginning }(\mathbf{g})\end{array}$ & $\begin{array}{c}\text { Weight at } \\
\text { the end }(\mathbf{g})\end{array}$ & $\begin{array}{c}\text { LH } \\
(\mathbf{n g} / \mathbf{d l})\end{array}$ & $\begin{array}{c}\text { FSH } \\
(\mathbf{n g} / \mathbf{d l})\end{array}$ & $\begin{array}{c}\text { Testosterone } \\
(\mathbf{m I U} / \mathbf{m l})\end{array}$ \\
\hline (I) Control & $152.37 \pm 10.04$ & $199.25 \pm 13.54$ & $23.00 \pm 1.90$ & $15.80 \pm 1.30$ & $0.40 \pm 0.09$ \\
(II) Extract $(250 \mathrm{mg} / \mathrm{kg})$ & $158.00 \pm 12.66$ & $207.87 \pm 17.23$ & $22.50 \pm 1.70$ & $13.41 \pm 2.04$ & $0.29 \pm 0.08$ \\
(III) Extract $(500 \mathrm{mg} / \mathrm{kg})$ & $160.37 \pm 13.00$ & $195.12 \pm 12.71$ & $22.90 \pm 1.9$ & $14.90 \pm 2.29$ & $0.30 \pm 0.06$ \\
(IV) PCOS & $158.12 \pm 9.17$ & $223.00 \pm 4.035^{*} \dagger$ & $24.70 \pm 1.50$ & $13.86 \pm 0.60$ & $3.70 \pm 0.90^{*}+\dagger$ \\
(V) PCOS + Extract $(250 \mathrm{mg} / \mathrm{kg})$ & $154.12 \pm 7.19$ & $210 \pm 19.27$ & $23.00 \pm 1.80$ & $13.23 \pm 0.43$ & $1.27 \pm 0.43$ \\
(VI) PCOS+ Extract $(500 \mathrm{mg} / \mathrm{kg})$ & $151.75 \pm 5.99$ & $190.25 \pm 17.63$ & $22.40 \pm 1.40$ & $14.90 \pm 1.20$ & $1.05 \pm 0.2$
\end{tabular}

Data are shown as mean \pm SD. P $\leq 0.05$ is considered statistically significant. *: Significant differences between PCOS-induced group (IV) and control group. $\uparrow$ : Significant differences between PCOS-induced group (IV) and group VI. $\uparrow \uparrow$ : Statistically significant differences between PCOS-induced group (IV) and groups V and VI.

Table 2. The number of primordial, primary, secondary, Graafian and atretic follicles, corpus lutea and cysts in ovarian tissue of the studied groups

\begin{tabular}{|c|c|c|c|c|c|c|c|}
\hline Groups & $\begin{array}{l}\text { Primordial } \\
\text { follicles }\end{array}$ & $\begin{array}{l}\text { Primary } \\
\text { follicles }\end{array}$ & $\begin{array}{l}\text { Secondary } \\
\text { follicles }\end{array}$ & $\begin{array}{c}\text { Graafian } \\
\text { follicles }\end{array}$ & $\begin{array}{l}\text { Atretic } \\
\text { follicles }\end{array}$ & $\begin{array}{l}\text { Corpus } \\
\text { luteum }\end{array}$ & $\begin{array}{c}\text { Ovarian } \\
\text { cysts }\end{array}$ \\
\hline (I) Control & $7.81 \pm 3.22 *, \oplus$ & $8.62 \pm 1.08$ & $5.56 \pm 1.09^{\S}$ & $0.31 \pm 0.40$ & $2.12 \pm 1.14^{* \dagger}$ & $5.93 \pm 1.52^{\dagger}$ & $0^{\dagger}$ \\
\hline (II) Extract (250 mg/kg) & $3.12 \pm 1.99$ & $7 \pm 2.06$ & $4.18 \pm 2.07$ & $0.25 \pm 0.44$ & $10.06 \pm 3.60$ & $4.93 \pm 1.84$ & 0 \\
\hline (III) Extract (500 mg/kg) & $0.87 \pm 0.61$ & $7.18 \pm 1.79$ & $4.65 \pm 2.06$ & $0.43 \pm 0.72$ & $7.18 \pm 4.47$ & $4.37 \pm 1.70$ & 0 \\
\hline (IV) PCOS & $2.93 \pm 1.34^{\dagger}$ & $7.37 \pm 1.50$ & $3.31 \pm 1.50$ & 0 & $12.56 \pm 1.96^{\bullet}$ & $1.31 \pm 1.07^{\bullet}$ & $10.18 \pm 3.01^{\bullet}$ \\
\hline (V) PCOS + Extract (250 mg/kg) & $2.12 \pm 1.08$ & $6.43 \pm 1.78$ & $2.25 \pm 1.73$ & 0 & $7.93 \pm 2.73$ & $4.06 \pm 1.98$ & $0.18 \pm 0.54$ \\
\hline (VI) PCOS + Extract $(500 \mathrm{mg} / \mathrm{kg})$ & $2.18 \pm 2.1$ & $8.00 \pm 1.15$ & $2.5 \pm 1.54$ & 0 & $7.56 \pm 2.73$ & $5.18 \pm 1.79$ & $0.12 \pm 0.34$ \\
\hline
\end{tabular}

Data are shown as mean \pm SD. $\mathrm{P} \leq 0.05$ is considered statistically significant. *: Significant differences between group II, III and control group. ${ }^{\dagger}$ Significant differences between PCOS-induced group (IV) and control group. ${ }^{\dagger}$ : Significant differences between control and groups V and VI. ${ }^{\S}$ : Significant differences between control and groups IV, V and VI. $\bullet$ : Significant differences between PCOS-induced group (IV) and groups V and VI. 

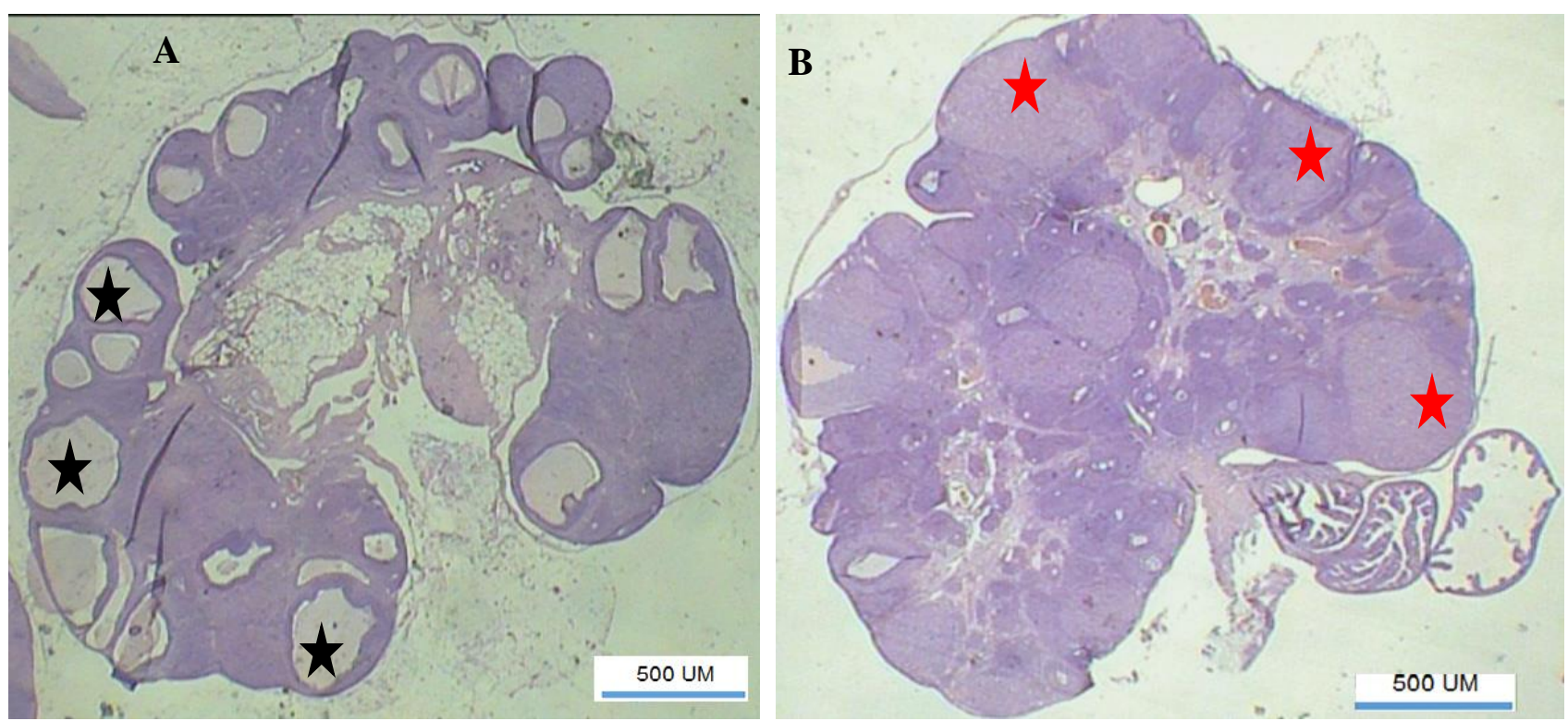

Figure 1. Light photomicrograph of ovarian tissue of the rats. Hematoxylin and eosin staining, 40x magnification. A: Ovarian tissue of polycystic ovary syndrome rats displays a considerable number of ovarian cysts. B: Ovarian tissue of polycystic ovary syndrome rats received spearmint extract $(500 \mathrm{mg} / \mathrm{kg})$ demonstrates no ovarian cyst. Treatment with spearmint extract has increased corpora lutea in this group compared with the polycystic ovary syndrome group. The red stars show corpora lutea, and the black stars show cystic follicles.

\section{DISCUSSION}

Nowadays, infertility is an important major concern of many couples which affects both men and women. PCOS is a condition that affects a woman's hormone levels that may lead to infertility related problems. Women with PCOS tend to have higher levels of androgens (Akdogan et al., 2007; Alaee et al., 2019). According to anti-androgenic effects of Mentha spicata and its beneficial effect in women with mild hirsutism and PCOS (Akdogan et al., 2007; Grant, 2010), in the current study, spearmint extract was administered to an animal model of PCOS to determine the effects of this herbal plant on LH, FSH and testosterone hormone levels, and also on folliculogenesis of ovaries. In addition, the effects of the extract on mentioned parameters were studied in normal rats. Measurement of body weight changes was one of the established methods to evaluate the toxicity of plant extracts (Gupta and Sharma, 2006). Since there was no significant change in the body weight of normal rats after administration of spearmint extract, it seemed that this medicinal plant has no general toxicity effect, which was also confirmed in other studies (Nozhat et al., 2004; Sadeghi et al., 2017). Furthermore, the spearmint at the level of administered doses had no effect on LH, FSH and testosterone levels in normal rats. Given the significant increase in the number of atretic follicles in normal rats which received spearmint extract, this agent may have detrimental effects on the ovarian folliculogenesis. Other studies have also demonstrated detrimental effects of spearmint tea on uterine, kidney and liver (Akdogan et al., 2003; Akdogan et al., 2004; Guney et al., 2006). Androgens are essential drivers of early and intermediate stages of follicular maturation. Locally produced androgens facilitate follicular development and serve as a substrate for estrogen production in the later stages of folliculogenesis (Pan et al., 2015). The optimum level of this hormone is crucial, because an excess level of androgens overrides follicular development, resulting in follicular arrest, follicular atresia and disturbance of ovulation (Gleicher et al., 2011).Therefore, the higher number of atretic follicles may be attributed to anti-androgenic effects of spearmint, but it should be determined whether the levels of testosterone and the number of corpus lutea, which are a manifestation of ovulation, did not decrease in the normal rats receiving spearmint extract. The disruption of follicular development in these groups may be related to the alteration in the level of other kinds of androgens that were not evaluated in our study, such as dihydrotestosterone and androstenedione, which are also involved in the growth and development of ovarian follicles in mammals (Cupisti et al., 2008, Lebbe and Woodruff, 2013). PCOS is a metabolic disease usually accompanied by insulin resistance, visceral obesity and elevated body mass index, all of which are correlated with an elevated level of oxidative stress and androgen production of the ovaries and adrenal glands, thus it contributes to the disturbed follicular development, oocyte maturation, and, ultimately, infertility (Shaikh et al., 2014b; Hussain et al., 2015; Papalou et al., 2016). The results showed that administration of spearmint extract to PCOS rats significantly reduces body weight and testosterone level.

In the present study, the weight did not change in normal rats that received spearmint extract. Current study found that spearmint has no effect on body weight in a normal condition. However, in PCOS condition, it may control and sustain body weight which may occur thorough metabolic mechanisms that were disturbed in the condition of PCOS. 
Therefore, spearmint extract may initiate a complicated mechanism that results in control of body weight, and also reduction of testosterone. It was demonstrated that spearmint leaves decrease cholesterol, and in type II diabetes, decrease oxidative stress, and improve activity of antioxidant enzymes (Rajeshwari et al., 2012). Al-Rekabi (2015) showed that administration of phenolic compounds of Mentha spicata leaves extract to diabetic male rats ca significantly enhance the antioxidant defense system, and reduce body weight and levels of glucose and cholesterol. Grant (2010) showed that administration of spearmint tea for 30 days significantly reduced free and total testosterone in PCOS women. A significant decrease in free testosterone level was also observed in women with hirsutism after receiving spearmint teas (Akdogan et al., 2007). It was shown that in the PCOS condition, ovarian steroidogenic enzyme deficiencies, such as aromatase deficiency, induced a hyperandrogenemic environment in the ovary, contributing to follicular maturation arrest and oligoovulation or anovulation (Rajeshwari et al., 2012). Reducing the body weight of anovulatory obese women decreased testosterone concentration, restored ovulation, and improved menstrual function and conception rates (Moran et al., 2003). However, in this study, although administration of spearmint led to a significant decrease in the body weight and testosterone level of PCOS-induced rats, the attenuated number of follicles caused by PCOS induction was not improved. Yet, similar to an identical study in which the effects of spearmint oil on PCOS were evaluated, the number of corpus luteum increased after the spearmint extract was administrated, reflecting the higher rate of ovulation in this group (Sadeghi et al., 2017). The high number of atretic follicles and ovarian cysts observed in PCOS-induced rats was thought to be associated with a high level of androgen. In PCOS animals that took spearmint extract, the number of atretic follicles and ovarian cysts decreased considerably, which could be associated with the anti-oxidant and anti-androgenic effects of the spearmint.

\section{CONCLUSION}

Spearmint as an anti-androgenic herb is believed to reduce testosterone level in PCOS condition, resulting in destruction of ovarian cysts and restoration of ovulation. It is suggested for future studies to evaluate ovarian antioxidant capacity and apoptosis status, and also fertility potential of PCOS-induced female rats after receiving spearmint extract at the level of mentioned dose used in this study.

\section{DECLARATIONS}

\section{Acknowledgments}

The authors of present article thank the Student Research Committee, Shiraz University of Medical Sciences, for the ethical approval and financial support of this research (grant number: 1396-01-21-15581).

\section{Competing interests}

The authors declare that there are no conflicts of interest.

\section{REFERENCES}

Akdogan M, Ozguner M, Aydin G and Gokalp O (2004). Investigation of biochemical and histopathological effects of Mentha piperita Labiatae and Mentha spicata Labiatae on liver tissue in rats. Human and Experimental Toxicology, 23 (1): 21-28. DOI: http://www.dx.doi.org/10.1191/0960327104ht412oa

Akdogan M, Tamer MN, Cüre E, Cüre MC, Köroğlu BK and Delibaş N (2007). Effect of spearmint (Mentha spicata Labiatae) teas on androgen levels in women with hirsutism. Phytotherapy Research, 21 (5): 444-447. DOI: https://www.doi.org/10.1002/ptr.2074

Akdogan M, Wnc I, Oncu M, Karaoz E and Delibas N (2003). Investigation of biochemical and histopathological effects of Mentha piperita L. and Mentha spicata L. on kidney tissue in rats. Human and Experimental Toxicology, 22 (4): 213-219. DOI: http://www.dx.doi.org/10.1191/0960327103ht332oa

Alaee S, Ghaffari Novin M, Noroozian M, Yeganeh F, Pakravesh J, Heidari MH and Salehpour S (2014). Evaluation of progesterone receptor, FKBP51 and FKBP52, associated with uterine receptivity, in endometrial tissue of women with repeated implantation failure. Acta Endocrinologica (Buc), 10 (3): 329-339. DOI: http://www.dx.doi.org/10.4183/aeb.2014.329

Alaee S, Rezaee S and Ziaei Gh (2016). Evaluation of the Effects of Mentha Spicata Extract on In-Vitro Maturation of Mouse Oocytes. Journal of Advanced Medical Sciences and Applied Technologies, 2 (2): 200-203. DOI: http://www.dx.doi.org/10.18869/NRIP.JAMSAT.2.2.200

Alaee S, Yousefian E, Talaiekhozani A, Ziaee GR and Homayoon H (2019). Infertility Knowledge, Attitudes, and Beliefs among Iranian College Students. Journal of Environmental Treatment Techniques, 7 (1): 171-178.

Al-Rekabi EA (2015). Anti-oxidant and hepatoprotective activity of phenolic compounds of leaves extracts from Mentha longifolia and Mentha spicata in diabetic male rats. World Journal of Pharmceutical Research, 4 (6): 346-354.

Cupisti S, Kajaia N, Dittrich R, Duezenli H, Beckmann MW and Mueller A (2008). Body mass index and ovarian function are associated with endocrine and metabolic abnormalities in women with hyperandrogenic syndrome. European Journal of Endocrinology, 158 (5): $711-719$. DOI: http://www.dx.doi.org/10.1530/eje-07-0515

Gleicher N, Weghofer A and Barad DH (2011). The role of androgens in follicle maturation and ovulation induction: friend or foe of infertility treatment? Reproductive Biology and Endocrinology, 9: 116. DOI: http://www.dx.doi.org/10.1186/1477-7827-9-116

Grant P (2010). Spearmint herbal tea has significant anti-androgen effects in polycystic ovarian syndrome a randomized controlled trial. Phytotherapy Research, 24 (2): 186-168. DOI: http://www.dx.doi.org/10.1002/ptr.2900 
Guimarães R, Barreira J, Barros L, Carvalho AM and Ferreira IC (2011). Effects of oral dosage form and storage period on the antioxidant properties of four species used in traditional herbal medicine. Phytotherapy Research, 25 (4): 484-892. DOI: http://www.dx.doi.org/10.1002/ptr.3284

Guney M, Oral B, Karahanli N, Mungan T and Akdogan M (2006). The effect of Mentha spicata Labiatae on uterine tissue in rats. Toxicological Industrial Health, 22 (8): 343-348. DOI: http://www.dx.doi.org/10.1177/0748233706071738

Gupta RS and Sharma R (2006). A review on medicinal plants exhibiting antifertility activity in males. Natural Product Radiance, 5 (5): $389-410$. Available at: https://pdfs.semanticscholar.org/831d/51c634ea2a9ebaff62d8ed0a95e5d83da200.pdf

Hatirnaz S, Gençdal S, Atasever M, Ekmekçi E, Bakacak M and Kelekçi S (2015). Evaluation of polycystic ovary syndrome patients treated for OHSS. Journal of Infertility and Reproductive Biology, 3 (2): 179-183. Available at: Link

Hussain M, Yehia AH, Abdelazim IA, Elbiaa A, Abdelrazak KM and Farghali MM (2015). Early clomiphene citrate for ovulation induction in polycystic ovary syndrome. Journal of Infertility and Reproductive Biology, 3 (4): 245-249. Available at: Link

Krishnapillai K, Nambiar D and Fency JL (2015). Comparison of ovarian response between PCOS and Non-PCOS patients undergoing ICSI with antagonist protocol Journal of Infertility and Reproductive Biology, 3 (3): 199-207. Available at: Link

Lebbe M and Woodruff T (2013). Involvement of androgens in ovarian health and disease. Molecular Human Reproduction, 19 (12): 828-837. DOI: http:// www.dx.doi.org/10.1093/molehr/gat065

Monsefi M and Masudi M (2014). Histomorphometrical changes of rat uterine and ovarian tissues fed chloroform fraction of dill (Anethum graveolens L.) extracts. Journal of Infertility and Reproductive Biology, 2 (1): 23-29. Available at: Link

Monsefi M, Ghasemi A, Alaee S and Aliabadi E (2015). Effects of Anethum graveolens L. (dill) on Oocyte and Fertility of Adult Female Rats. Journal of Reproduction and Infertility, 16 (1): 10-17. Available at: https://www.ncbi.nlm.nih.gov/pmc/articles/PMC4322175/

Monsefi M, Hosseini E and Alaee S (2013). Lectin Histochemical Study of Rat Reproductive Tissues Treated with Ether Fraction of Anethum graveolens L. Extracts. Anatomical Sciences Journal, $10 \quad$ (4): http://anatomyjournal.ir/browse.php?a_id=58\&sid=1\&slc_lang=en

Moran L, Noakes M, Clifton P, Tomlinson Land Norman R (2003). Dietary composition in restoring reproductive and metabolic physiology in overweight women with polycystic ovary syndrome. Journal of Clinical Endocrinology \& Metabolism, 88 (2): 812-819. DOI: http:// www.dx.doi.org/10.1210/jc.2002-020815

Namavar Jahromi B, Farrokhnia F, Tanideh N, Vijayananda Kumar P, Parsanezhad ME and Alaee S (2019). Comparing the effects of glycyrrhiza glabra root extract, a cyclooxygenase-2 inhibitor (celecoxib) and a gonadotropin-releasing hormone analog (diphereline) in a rat model of endometriosis. International Journal of Fertility and Sterility, 13 (1): 45-50. DOI: http://www.dx.doi.org/10.22074/ijfs.2019.5446

Neisy A, Zal F, Seghatoleslam A and Alaee S (2019). Amelioration by quercetin of insulin resistance and uterine GLUT4 and ER $\alpha$ gene expression in rats with polycystic ovary syndrome (PCOS). Reproduction, Fertility and Development, 31 (2): 315-323. DOI: http://www.dx.doi.org/10.1071/RD18222

Nozhat F, Alaee S, Behzadi K and Chegini NA (2014). Evaluation of possible toxic effects of spearmint (Mentha spicata) on the reproductive system, fertility and number of offspring in adult male rats. Avicenna Journal of Phytomedicine, 4 (6): 420-429. Available at: https://pubmed.ncbi.nlm.nih.gov/25386406/

Pan J-X, Zhang J-Y, Ke Z-H, Wang F-F, Barry JA, Hardiman PJ and Qu F (2015). Androgens as double-edged swords: Induction and suppression of follicular development. Hormones, 14 (2): 190-200. DOI: http://www.dx.doi.org/10.14310/horm.2002.1580

Papalou O, M Victor V and Diamanti-Kandarakis E (2016). Oxidative stress in polycystic ovary syndrome. Current Pharmaceutical Design, 22 (18): 2709-2722. DOI: http://www.dx.doi.org/10.2174/138161282266616021615185

Rajeshwari C, Preeti M and Andallu B (2012). Efficacy of mint (Mentha spicata L.) leaves in combating oxidative stress in type 2 diabetes. International Journal of Life Sciences, 1 (1): 28-34. Available at: http://www.crdeepjournal.org/wp-content/uploads/2012/10/1.IJLS-Vol.pdf

Sadeghi Ataabadi M, Alaee S, Bagheri MJ and Bahmanpoor S (2017). Role of Essential Oil of Mentha Spicata (Spearmint) in Addressing Reverse Hormonal and Folliculogenesis Disturbances in a Polycystic Ovarian Syndrome in a Rat Model. Advanced Pharmaceutical Bulletin, 7 (4): 651 654. DOI: http://www.dx.doi.org/10.15171/apb.2017.078

Shaikh N, Shinde GB and Nath N (2014a). Relevance of serum ascorbic acid status in ovulation and pregnancy outcome of non-PCOS women undergoing intrauterine insemination cycles. Journal of Infertility and Reproductive Biology, 2 (2): 43-49. Avialable at: Link

Shaikh N, Shinde GB and Nath N (2014b). The status of ascorbic acid in follicular fluid of non-PCOS women during IVF-ET cycles is an indicator for aromatization andpregnancy outcome. Journal of Infertility and Reproductive Biology, 2 (2): 56-61. Available at: Link

Sharangouda J, Patil, Venkatesh S, Vishwanatha T and Patil SB (2015). Effect of isolated chromatographic fractions of Citrus medica seeds: In vivo study on anti-implantation and estrogenic activity in albino rats. Journal of Infertility and Reproductive Biology, 3 (1): 136-144. Available at: $\underline{\text { Link }}$

Van Houten EL, and Visser JA (2014). Mouse models to study polycystic ovary syndrome: a possible link between metabolism and ovarian function? Reproductive Biology, 14 (1): 32-43. DOI: http://dx.doi.org/10.1016/j.repbio.2013.09.007

Vejdani R, Shalmani HR, Mir-Fattahi M, Sajed-Nia F, Abdollahi M, Zali MR, Alizadeh AH, Bahari A and Amin G (2006). The efficacy of an herbal medicine, Carmint, on the relief of abdominal pain and bloating in patients with irritable bowel syndrome: a pilot study. Digestive Diseases and Sciences, 51 (8): 1501-1507. DOI: http://www.dx.doi.org/10.1007/s10620-006-9079-3 